\title{
Epithelial-mesenchymal transition confers resistance to selective FGFR inhibitors in SNU-16 gastric cancer cells
}

\author{
Paulina Grygielewicz • Barbara Dymek • Anna Bujak • Pawel Gunerka • \\ Aleksandra Stanczak • Monika Lamparska-Przybysz • Maciej Wieczorek • \\ Karolina Dzwonek • Daria Zdzalik
}

Received: 24 June 2014 / Accepted: 1 November 2014/Published online: 19 November 2014

(C) The Author(s) 2014. This article is published with open access at Springerlink.com

\begin{abstract}
Background Up to $10 \%$ of primary gastric cancers are characterized by $F G F R 2$ amplification, and fibroblast growth factor receptor (FGFR) inhibitors may represent therapeutic agents for patients with these malignancies. However, long-term benefits of the treatment might be limited owing to the occurrence of drug resistance.

Methods To investigate the mechanisms of resistance to selective FGFR inhibitors, we established three FGFR2amplified SNU-16 gastric cancer cell lines resistant to AZD4547, BGJ398, and PD173074, respectively.

Results The resistant cell lines (SNU-16R) demonstrated changes characteristic of epithelial-to-mesenchymal transition (EMT). In addition, they displayed loss of expression of FGFR2 and other tyrosine kinase receptors concurrent
\end{abstract}

Electronic supplementary material The online version of this article (doi:10.1007/s10120-014-0444-1) contains supplementary material, which is available to authorized users.

P. Grygielewicz · B. Dymek · A. Bujak · P. Gunerka ·

A. Stanczak · M. Lamparska-Przybysz · M. Wieczorek ·

K. Dzwonek · D. Zdzalik

Innovative Drugs R\&D Department, Celon Pharma Inc.,

Mokra 41a, 05-092 Lomianki/Kielpin, Poland

P. Grygielewicz $(\bowtie) \cdot$ A. Bujak

Postgraduate School of Molecular Medicine,

Zwirki i Wigury 61, 02-091 Warsaw, Poland

e-mail: paulina.grygielewicz@celonpharma.com

P. Gunerka

Department of Medical Biotechnology, Medical

University of Lodz, Al. Kosciuszki 4, 90-419 Łodz, Poland

\section{K. Dzwonek}

Department of Immunology, Center for Biostructure Research,

Medical University of Warsaw, Banacha 1a, F Building,

02-097 Warsaw, Poland with activation of downstream signaling proteins and upregulation of the transforming growth factor $\beta$ (TGF- $\beta$ ) level. However, treatment of parental SNU-16 cells with TGF- $\beta_{1}$ did not evoke EMT, and pharmacological inhibition of TGF- $\beta$ receptor I was not sufficient to reverse EMT changes in the resistant cells. Finally, we showed that the SNU-16R cell lines were sensitive to the human epidermal growth factor receptor 2 inhibitor mubritinib and the heat shock protein 90 inhibitor AUY922.

Conclusion In conclusion, we provide experimental evidence that EMT-mediated resistance might emerge in gastric cancer patients following treatment with FGFR inhibitors, and mubritinib or AUY922 treatment may be an alternative therapeutic strategy for these patients.

Keywords Fibroblast growth factor receptor - Epithelialmesenchymal transition - Drug resistance $\cdot$ Fibroblast growth factor receptor inhibitor · AZD4547 · BGJ398

\section{Introduction}

The fibroblast growth factor (FGF) receptors (FGFRs) constitute one of the most extensively studied novel therapeutic targets in the field of anticancer drug development. The FGFR family comprises four members (FGFR1FGFR4) which serve as high-affinity receptors for 22 different FGF ligands [1]. The FGF/FGFR pathway plays an important role in a myriad of key cellular processes, such as cell proliferation, differentiation, migration, survival, and angiogenesis [1]. Aberrant activation of FGFR kinases owing to gene amplification, chromosomal translocation, or gain-of-function mutation has been implicated in the pathogenesis of a variety of human cancers, including gastric cancer [2], breast cancer [3], bladder cancer [4], 
endometrial cancer [5], squamous cell lung cancer [6], multiple myeloma [7], and glioblastoma [8].

Since aberrantly activated FGFR kinases serve as an oncogenic "driver," a great number of FGFR inhibitors are currently in clinical development. However, the vast majority of them are multikinase inhibitors such as dovitinib, nintedanib, or ponatinib [9]. To date, no selective small-molecule FGFR inhibitor has been approved for clinical use. The first selective FGFR inhibitor developed was PD173074, which, despite its high selectivity and cellular activity, has never entered clinical use [10]. At present, only a few selective FGFR inhibitors are under clinical investigation, and the two in the most advanced stage are AZD4547 and BGJ398, developed by AstraZeneca and Novartis, respectively [11, 12].

According to the World Health Organization, gastric cancer accounts for nearly one in ten of all deaths of cancer patients [13]. Mean survival for patients with stage IV metastatic gastric cancer is only 10 months [14]. Given this, there is an urgent need to improve gastric cancer therapy. FGFR2 amplification occurs in 3-10\% of primary gastric cancers, and has been reported to be more frequent in the undifferentiated diffuse subtype [2]. The effectiveness of FGFR inhibitors against gastric cancer in vitro and in vivo suggests that the use of these compounds may be a therapeutic strategy for the selected group of patients with FGFR2-amplified gastric cancer [15, 16]. However, from experience with other kinase inhibitors used in clinical practice, there is a high probability that resistance will develop in patients following FGFR inhibitor treatment.

To date, there is a lack of clinical data concerning drug resistance in FGFR-inhibitor-based therapy. Nevertheless, recently published results from in vitro models cast some light on the possible mechanisms of resistance to FGFR inhibitors. It has been shown that the heterozygous gatekeeper mutation FGFR3 V555M emerged as a mechanism of acquired resistance to the selective FGFR inhibitor AZ12908010 in KMS11 myeloma cells, and the resistant cells were cross-resistant to other FGFR inhibitors, AZD4547 and PD173074 [17]. Additionally, several activating mutations were identified in FGFR2-expressing $\mathrm{Ba} / \mathrm{F} 3$ cells treated with high concentrations of dovitinib. Recognized mutations conferred crossresistance to the selective FGFR inhibitor PD173074 but not to the multikinase inhibitor ponatinib, whose inhibitory activity was affected only by the V565I gatekeeper mutation [18]. In addition to secondary mutations in the kinase domain, resistance to kinase inhibitors may also occur through activation of alternative signaling pathways. With use of a highthroughput "secretome" screening platform, it has been established that ligand-mediated activation of compensatory kinases such as epidermal growth factor receptor (EGFR), human EGFR (HER), or MET may compensate for loss of FGFR kinase activity [19]. Additionally, using parallel RNA interference genetic screens, Herrera-Abreu et al. [20] discovered that EGFR is a key mediator of resistance in FGFR3dependent cancer cells.

Given this, to predict the mechanisms of acquired resistance which may emerge in patients with FGFR2dependent gastric cancer following treatment with selective FGFR inhibitors, we generated three SNU-16 cell lines resistant (SNU-16R) to AZD4547, BGJ398, and PD173074, respectively. It has been well established that SNU-16 cells harbor FGFR2 amplification, and thereby they are highly sensitive to FGFR inhibitors in vitro and in vivo [2, 21-23]. However, long-term exposure to increasing concentrations of selected inhibitors induced resistance accompanied by loss of FGFR2 expression and epithelial-to-mesenchymal transition (EMT), a phenomenon that has previously been shown to be involved in resistance to other kinase inhibitors [24-27]. Finally, on the basis of our results, we propose possible therapeutic strategies to overcome EMT-mediated resistance to FGFR inhibitors.

\section{Materials and methods}

Compounds and cell lines

AT9283, AZD4547, AZD6244, BGJ398, crizotinib, pictilisib, ibrutinib, PD173074, ruxolitinib, TAE684, and gandotinib were all purchased from Selleck Chemicals, AUY922, dovitinib, everolimus, gefitinib, mubritinib, saracatinib, sunitinib, and ZSTK474 were from LC Laboratories, CH5424802 was from Active Biochem, SB525334 was from Tocris, and fedratinib was from Axon Medchem. The human gastric cancer cell line SNU-16 was obtained from ATCC and was cultured in RPMI 1640 medium supplemented with $10 \%$ fetal bovine serum according to the manufacturer's instructions.

Generation of AZD4547-, BGJ398-, and PD173074resistant SNU-16 cells

To generate drug-resistant cells, SNU-16 cells were exposed to increasing concentrations of AZD4547, BGJ398, or PD173074 for 1 month. The concentration of each inhibitor was doubled at every second passage to a final concentration of $0.6 \mu \mathrm{M}$ for AZD4547 and $1.2 \mu \mathrm{M}$ for BGJ398 and PD173074. The resistant cells were cultured until they again had growth kinetics similar to the growth kinetics of untreated parental cells.

Treatment of parental SNU-16 cells with transforming growth factor $\beta_{1}$

SNU-16 cells were cultured in a medium containing human recombinant transforming growth factor (TGF)- $\beta_{1}$ (Merck 
Millipore) in a final concentration of $0.5 \mathrm{ng} / \mathrm{ml}$. Fresh TGF- $\beta_{1}$ was added every $72 \mathrm{~h}$ for 3 weeks.

\section{Cell viability assays}

For viability tests, $6 \times 10^{3}$ cells per well were seeded in 96-well plates and exposed to increasing doses of the tested compounds. After $72 \mathrm{~h}$ of drug treatment, cell viability was determined by the ATPlite assay (Perkin Elmer) according to the manufacturer's instructions.

Half maximal inhibitory concentrations $\left(\mathrm{IC}_{50}\right)$ of mubritinib were calculated with GraphPad Prism using the sigmoid dose-response function. All experiments were performed at least twice.

Immunoblot analysis

SNU-16 parental and resistant cells were seeded in six-well plates at a density of $0.5 \times 10^{6}$ cells per milliliter in an inhibitor-free medium. After $24 \mathrm{~h}$, cells were lysed and the level of proteins was examined by Western blotting according to the protocols provided by the antibody suppliers. The antibodies against MET, EGFR, HER2, extracellular-signal-regulated kinase (ERK), phosphorylated AKT, phosphorylated EGFR, phosphorylated ERK (pERK), phosphorylated FGFR, phosphorylated FGFR substrate, and phosphorylated signal transducer and activator of transcription (STAT3) were purchased from Cell Signaling Technology, $\beta$-tubulin and AKT were from Millipore, E-cadherin, STAT3, and $\beta$-catenin were from BD Biosciences, FGFR2 was from Abnova, and vimentin was from Calbiochem. All experiments were performed at least twice.

\section{Phosphoprotein array analysis}

SNU-16 parental and resistant cells were seeded in T25 bottles at a density of $1.5 \times 10^{6}$ cells per bottle in an inhibitor-free medium. The assay was conducted in accordance with the commercial protocol of the PathScan ${ }^{\circledR}$ receptor tyrosine kinase signaling antibody array kit (Cell Signaling Technology).

\section{Results}

SNU-16R cell lines exhibited a substantial decrease in sensitivity to all FGFR inhibitors tested

To generate SNU-16 gastric cancer cell lines resistant to AZD4547 (AZDR), BGJ398 (BGJR), and PD173074 (PDR), SNU-16 cells were cultured with increasing concentrations of the respective FGFR inhibitor. In the viability assay, the parental cell line was shown to be sensitive to FGFR inhibitors, with $50 \%$ inhibition of cell viability at a concentration of approximately $150 \mathrm{nM}$ for AZD4547 and BGJ398, and $380 \mathrm{nM}$ for PD173074 (Fig. 1). The observed growth inhibition was dose independent in range from 0.153 to $6 \mu \mathrm{M}$, and the viability of SNU-16 cells was completely inhibited only at high micromolar concentrations of the FGFR inhibitors. Given this, we were unable to fit sigmoid dose-response curves to the data and calculate true $\mathrm{IC}_{50}$ values. Consistent with the ability of resistant cells to grow at high concentrations of a particular FGFR inhibitor, AZDR, BGJR, and PDR cells were highly resistant to AZD4547, BGJ398, or PD173074, respectively, with an $\mathrm{IC}_{50}$ of approximately $10 \mu \mathrm{M}$ (Fig. 1). Moreover, each resistant cell line exhibited crossresistance to all FGFR inhibitors tested, and the resistance to AZD4547, BGJ398, and PD173074 was comparable among all resistant cell lines. Altogether, the substantial decrease in sensitivity of SNU-16R cell lines to FGFR inhibitors observed in the cell viability assay confirmed the emergence of drug resistance in SNU-16R cells.

SNU-16R cells lose their dependency on FGFR2 signaling and display alterations in other signaling pathways

To examine the mechanism of resistance to FGFR inhibitors in SNU-16R cell lines, firstly we determined the status of FGFR2 signaling by immunoblot analysis. It revealed that the level of FGFR2 was undetectable in resistant cells in contrast to parental ones (Fig. 2a). Consistent with these findings, the decrease of phosphorylation of phosphorylated FGFR substrate 2, a direct downstream target of FGFR2, was also observed (Fig. 2a). Given this, all SNU16R cell lines became independent of FGFR2 signaling. However, despite loss of FGFR2 expression, phosphorylation of downstream proteins such as ERK, AKT, and STAT3 was upregulated in AZDR, BGJR, and PDR cells (Fig. 2a). This suggested that activation of alternative pathways could be responsible for the observed increase in phosphorylation status of downstream proteins.

To verify whether acquired resistance of AZDR, BGJR, and PDR cells is the effect of the induction of an alternative signaling pathway, we used the slide-based PathScan ${ }^{\circledR}$ receptor tyrosine kinase signaling antibody array kit. Phosphoprotein array analysis confirmed that all SNU-16R cell lines exhibit alterations in diverse signaling pathways in comparison with parental SNU-16 cells. We found a slight increase in the phosphorylation status of SRC and RET concomitantly with downregulation of phosphorylated MET and phosphorylated HER3 in all three SNU-16R cell lines compared with wild-type SNU-16 cells (Fig. 2b). Additional immunoblot analysis showed that the decrease 
Fig. 1 Sensitivity of parental SNU-16 and resistant SNU-16 (SNU-16R) cells to selective fibroblast growth factor receptor (FGFR) inhibitors. Cells were treated with increasing concentrations of AZD4547, BGJ398, or PD173074 for $72 \mathrm{~h}$, and cell viability was measured with the ATPlite assay. Each data point represents the mean of two independent triplicate measurements, and error bars indicate the standard deviation. AZDR SNU-16 cell line resistant to AZD4547, BGJR SNU-16 cell line resistant to BGJ398, PDR SNU-16 cell line resistant to PD173074
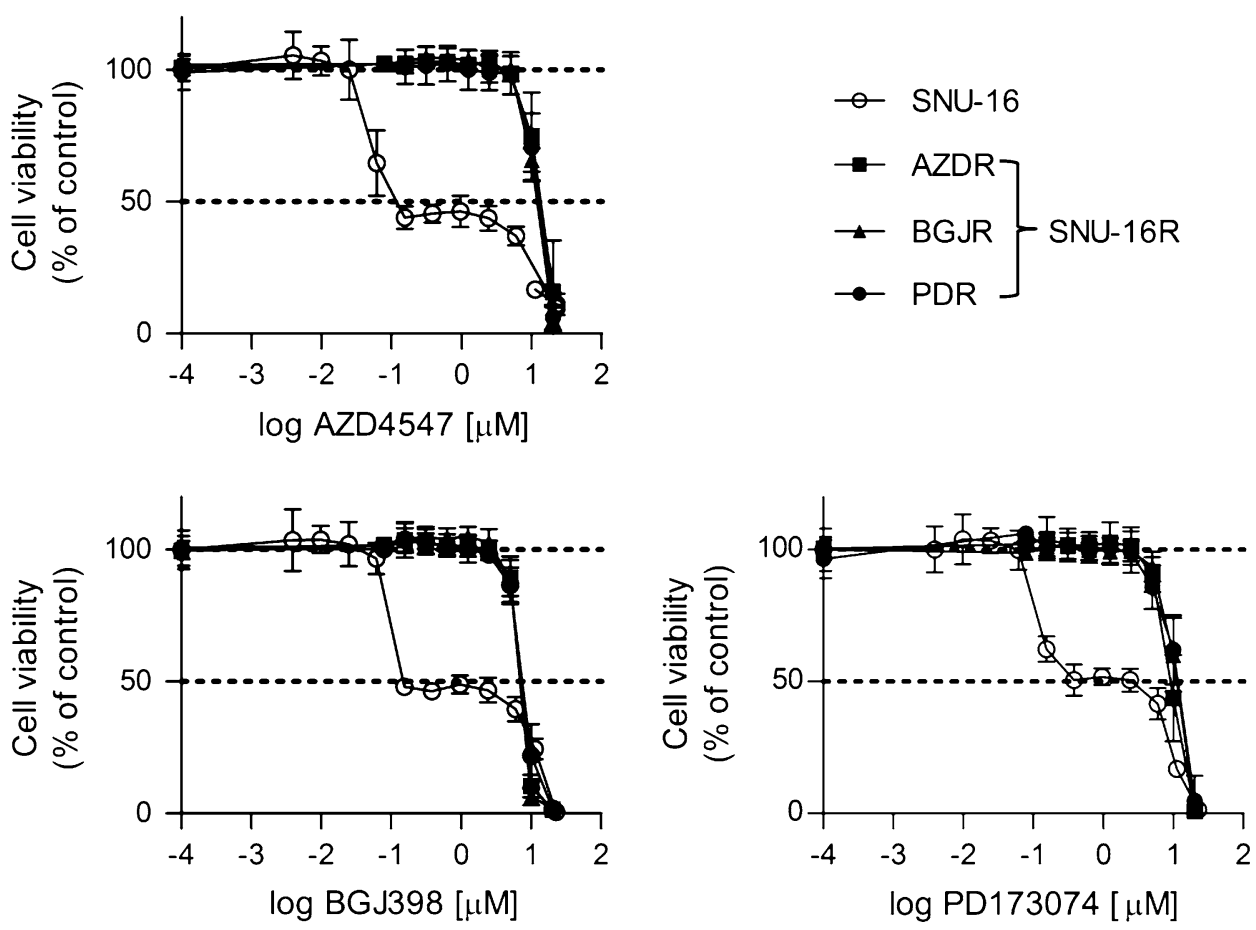

a

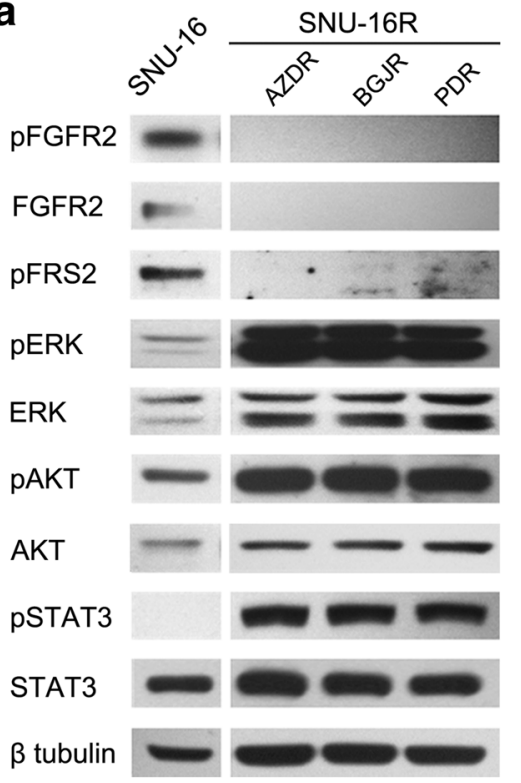

b

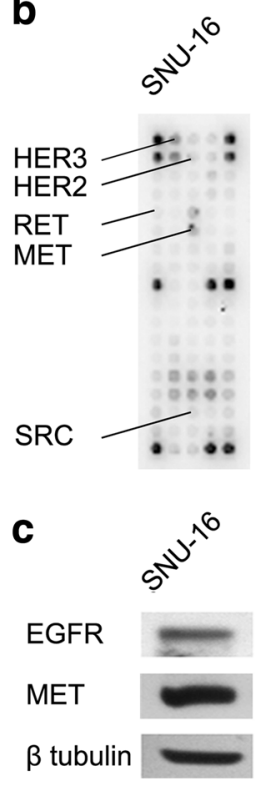

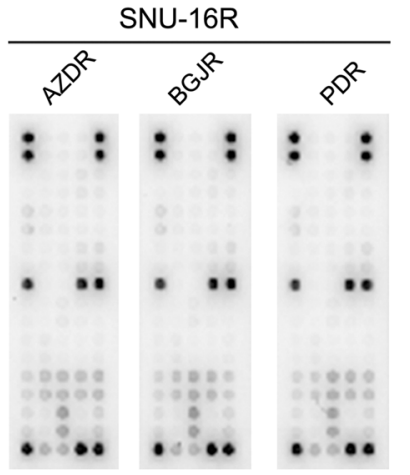

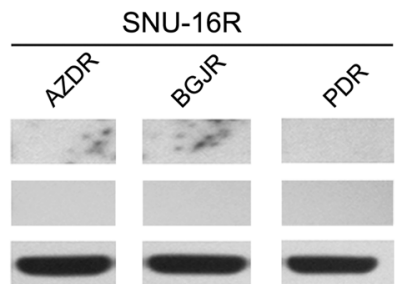

Fig. 2 Alterations in signaling pathways in resistant SNU-16 (SNU$16 R)$ cell lines. Parental SNU-16 and SNU-16R cells were incubated in inhibitor-free medium for $24 \mathrm{~h}$, and then cell lysates from each cell line were subjected to immunoblot analysis. A representative result from one of two separate experiments is shown. a Immunoblot analysis of FGFR-dependent signaling and downstream proteins. b Analysis of phosphorylation status of key signaling molecules assessed by the PathScan ${ }^{\circledR}$ receptor tyrosine kinase signaling antibody array. c Immunoblot analysis of the expression of the receptors epidermal growth factor receptor (EGFR) and MET. AZDR SNU-16 cell line resistant to AZD4547, BGJR SNU-16 cell line resistant to BGJ398, ERK extracellular-signal-regulated kinase, FGFR fibroblast growth factor receptor, HER2 human epidermal growth factor receptor 2, HER3 human epidermal growth factor receptor 3, PDR SNU-16 cell line resistant to PD173074, $p E R K$ phosphorylated extracellular-signal-regulated kinase, $p F G F R$ phosphorylated fibroblast growth factor receptor, $p F R S 2$ phosphorylated fibroblast growth factor receptor substrate 2, pSTAT3 phosphorylated signal transducer and activator of transcription 3, STAT3 signal transducer and activator of transcription 3 
a
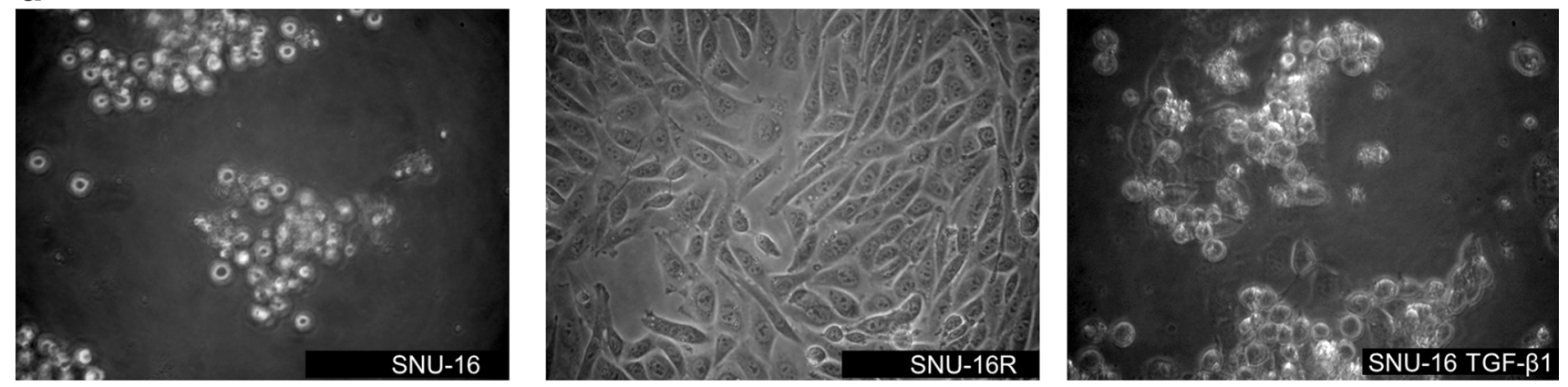

b

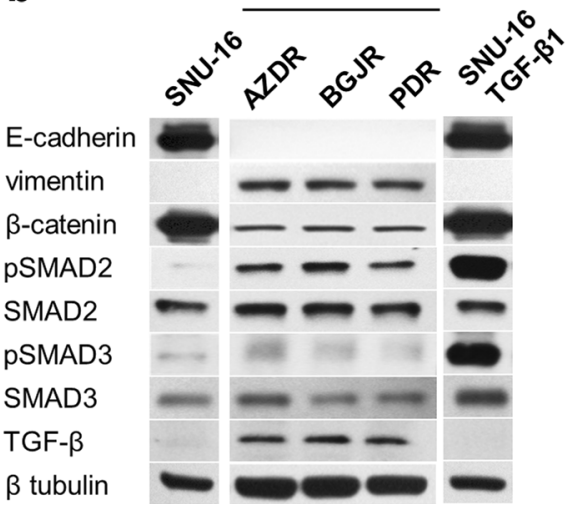

C

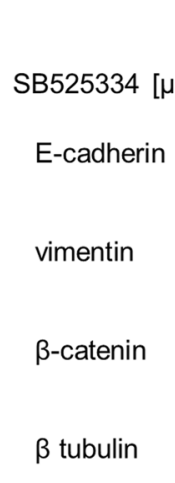

SNU-16

SNU-16R

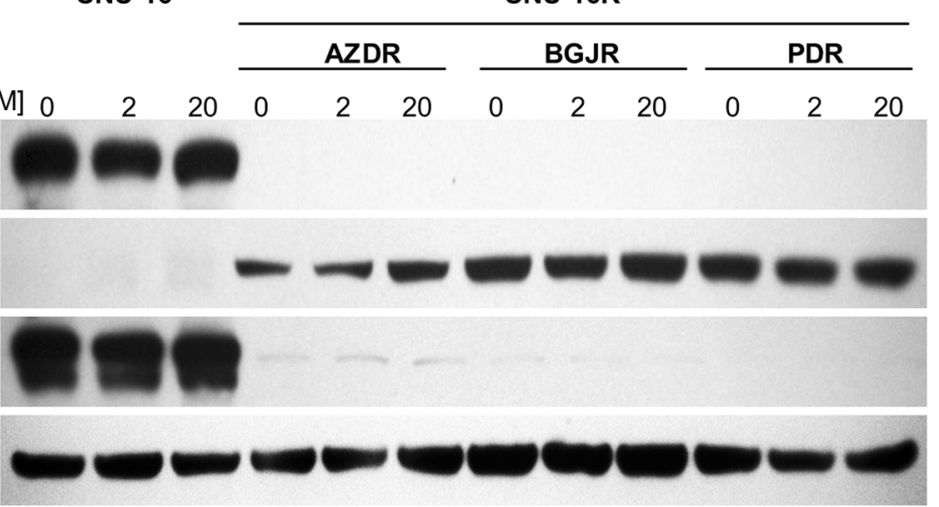

Fig. 3 Induction of epithelial-to-mesenchymal transition (EMT) in resistant SNU-16 (SNU-16R) cell lines. a The morphology of parental SNU-16 cells, SNU-16R cells, and SNU-16 cells treated with transforming growth factor $(T G F) \beta_{1}$ observed under a light microscope (magnification $\times 200$ ). b Immunoblot analysis of the expression of EMT markers and TGF- $\beta_{1}$ signaling proteins in parental SNU-16 cells and SNU-16R cells following incubation in inhibitor-

in the phosphorylated MET level was correlated with loss of total MET protein expression, and loss of other transmembrane proteins such as EGFR also occurred (Fig. 2c).

\section{EMT-like changes are induced in SNU-16R cell lines}

We observed a remarkable morphologic difference between parental SNU-16 and SNU-16R cell lines. As shown in Fig. 3a, SNU-16R cells acquired a fibroblastlike, spindle-shaped morphology, suggesting that resistant cells might have undergone EMT-like changes. Recently, growing evidence has suggested that EMT is implicated in resistance to kinase-inhibitor-based therapy [24-27]. Since EMT is characterized by downregulation of epithelial phenotype markers such as E-cadherin and upregulation of mesenchymal phenotype markers such as vimentin [28], we tested the level of these markers in AZDR, BGJR, and PDR cells. This analysis revealed that E-cadherin expression was significantly reduced and vimentin expression was upregulated in all SNU-16R cell lines when compared with the parental SNU-16 cells free medium for $24 \mathrm{~h}$ and in parental SNU-16 cells treated with TGF$\beta_{1}$ for 3 weeks. c Immunoblot analysis of the expression of EMT marker proteins in parental SNU-16 and SNU-16R cell lines following incubation with the TGF- $\beta$ receptor I inhibitor SB525334 for 24 h. $p S M A D 2$ phosphorylated SMAD2, $p S M A D 3$ phosphorylated SMAD3

(Fig. 3b). Additionally, we observed a substantial decrease in total $\beta$-catenin level in resistant cells, which was probably the result of acquisition of a mesenchymal phenotype associated with loss of E-cadherin $/ \beta$-cateninbased adherens junctions (Fig. 3b). However, we cannot exclude intranuclear accumulation of $\beta$-catenin, a wellknown EMT marker, in AZDR, BGJR, and PDR cells $[29,30]$. Taken together, our findings indicate that EMT characteristics accompanied the acquisition of resistance to AZD4547, BGJ398, and PD173074.

\section{SNU-16R cell lines have enhanced expression of TGF- $\beta$}

It has previously been shown that TGF- $\beta$ signaling plays an important role in EMT, and treatment of cells with TGF- $\beta_{1}$ can trigger EMT changes [24]. To verify the involvement of TGF- $\beta$ signaling in EMT changes in SNU-16R cell lines, we analyzed the expression of TGF- $\beta$ and activation of its downstream molecules SMAD2 and SMAD3. As shown in Fig. 3b, TGF- $\beta$ expression was upregulated in all 


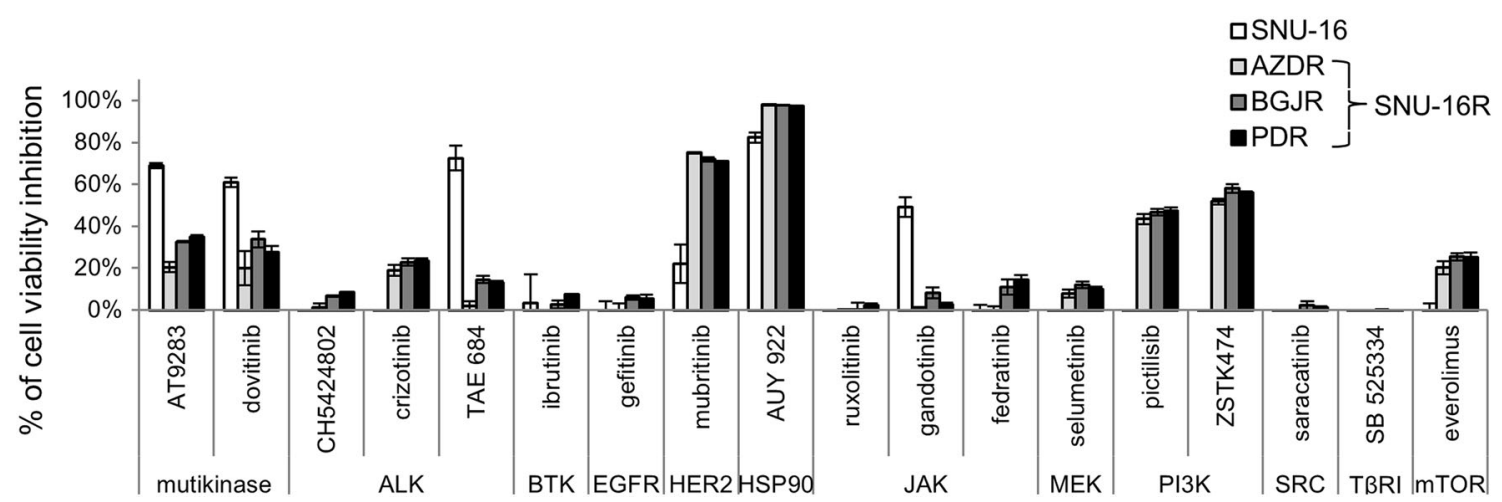

Fig. 4 Sensitivity of parental SNU-16 and resistant SNU-16 (SNU$16 R$ ) cell lines to different small-molecule inhibitors. Viability of parental SNU-16 and SNU-16R cell lines following $72 \mathrm{~h}$ of incubation with the indicated inhibitors at $1 \mu \mathrm{M}$ concentration. Each data point represents the mean of at least two independent experiments, and error bars indicate the standard deviation. ALK anaplastic lymphoma kinase, AZDR SNU-16 cell line resistant to AZD4547,

resistant SNU-16R cells, and this was accompanied by a slight increase in the phosphorylation of SMAD2.

SNU-16 cells treated with TGF- $\beta_{1}$ do not mimic EMT changes displayed in resistant cell lines

To evoke EMT in SNU-16 cells and mimic conditions which occur in in vivo tumor microenvironment [31] as well as to promote a sustained EMT and an invasive phenotype [32], cells were treated with TGF- $\beta_{1}$ for 3 weeks. As shown in Fig. 3b, TGF- $\beta_{1}$ treatment induced an increase in the phosphorylation level of SMAD2 and SMAD3 (Fig. 3b). However, despite observed activation of the TGF- $\beta$ signaling pathway, SNU-16 cells did not acquire characteristics associated with EMT, following 3 weeks of incubation with TGF- $\beta_{1}$ (Fig. 3a, b).

EMT characteristics of SNU-16R cell lines are not abrogated by inhibition of TGF- $\beta$ receptor I

Owing to the essential role of TGF- $\beta$ signaling in induction and maintaining EMT, TGF- $\beta$ signaling pathway inhibitors are able to reverse EMT [33]. Given this, in order to evoke induction of EMT in SNU-16 cells, we treated SNU-16R cell lines with SB525334 - an inhibitor of activin-receptorlike kinase 5, an isoform of TGF- $\beta$ receptor I (T $\beta R I$ ). However, $24 \mathrm{~h}$ incubation with SB525334 did not reverse EMT since we did not observe an increase in E-cadherin level and a decrease in vimentin level (Fig. 3c). Additionally, SB525334 treatment for $72 \mathrm{~h}$ did not have any impact on the viability of all SNU-16R and parental SNU16 cells (Fig. 4). Taken together, no EMT changes similar to those observed after long-term exposure to FGFR inhibitors were observed following TGF- $\beta_{1}$ treatment.
BGJR SNU-16 cell line resistant to BGJ398, BTK Bruton agammaglobulinemia tyrosine kinase, $E G F R$ epidermal growth factor receptor, HER2 human epidermal growth factor receptor 2, $H S P 90$ heat shock protein 90, JAK Janus kinase, mTOR mammalian target of rapamycin, $P D R$ SNU-16 cell line resistant to PD173074, PI3K phosphatidylinositol 3-kinase, TRRI transforming growth factor $\beta$ receptor I

Mubritinib and AUY922 overcome FGFR-inhibitorinduced resistance

To identify potential alternative drug therapies for gastric cancer with EMT-mediated resistance to FGFR inhibitors, we tested the sensitivity of SNU-16R and parental SNU-16 cells to several established and investigational anticancer agents. Initially, to evaluate their impact on cell growth, the cells were treated with selected inhibitors at a concentration of $1 \mu \mathrm{M}$ for $72 \mathrm{~h}$ (Fig. 4). Using this approach, we found that all SNU-16R cell lines remained sensitive to a similar extent as parental cells to the heat shock protein 90 (HSP90) inhibitor AUY922. Moreover, in contrast to the parental cell line, AZDR, BGJR, and PDR cells were shown to be more sensitive to pan-phosphatidylinositol 3-kinase inhibitors (pictilisib and ZSTK474) and a potent inhibitor of HER2 (mubritinib). However, ZSTK474 inhibited viability of parental and resistant cell lines with submicromolar $\mathrm{IC}_{50}$, with just slightly better efficacy against resistant cells (data not shown).

More significant from a therapeutic point of view is that mubritinib suppressed growth of the resistant cell lines at nanomolar concentrations and had little effect on the viability of the parental cell line (Fig. 4b). The calculated $\mathrm{IC}_{50}$ values for resistant cell lines were as follows: $146 \mathrm{nM}$ for AZDR cells, $298 \mathrm{nM}$ for BGJR cells, and $171 \mathrm{nM}$ for PDR cells. In the case of wild-type SNU-16 cells, the $\mathrm{IC}_{50}$ value was not calculated since sigmoid dose-response curves could not be fitted properly to the data obtained.

As all three resistant cell lines displayed the same changes, we examined the effects of mubritinib treatment on the phosphorylation status of downstream proteins in AZDR cells. As shown in Fig. 5b, mubritinib reduced the level of pERK in the AZDR cell line but not in wild-type 
Fig. 5 Resistant SNU-16 $(S N U-16 R)$ cell lines are sensitive to the human epidermal growth factor receptor 2 (HER2) inhibitor mubritinib. a Cell viability assay of a parental cell line and resistant cell lines following $72 \mathrm{~h}$ of incubation with increasing concentrations of mubritinib. Each data point represents the mean of two independent triplicate measurements, and error bars indicate the standard deviation. b Immunoblot analysis of protein phosphorylation status in parental SNU-16 cells and SNU-16 cells resistant to AZD4547 (ADZR) treated for $2 \mathrm{~h}$ with the indicated concentrations of mubritinib. $B G J R$ SNU-16 cell line resistant to BGJ398, ERK extracellularsignal-regulated kinase, $P D R$ SNU-16 cell line resistant to PD173074, $p E R K$

phosphorylated extracellularsignal-regulated kinase, $p H E R 2$ phosphorylated HER2, pSTAT3 phosphorylated signal transducer and activator of transcription 3, STAT3 signal transducer and activator of transcription 3
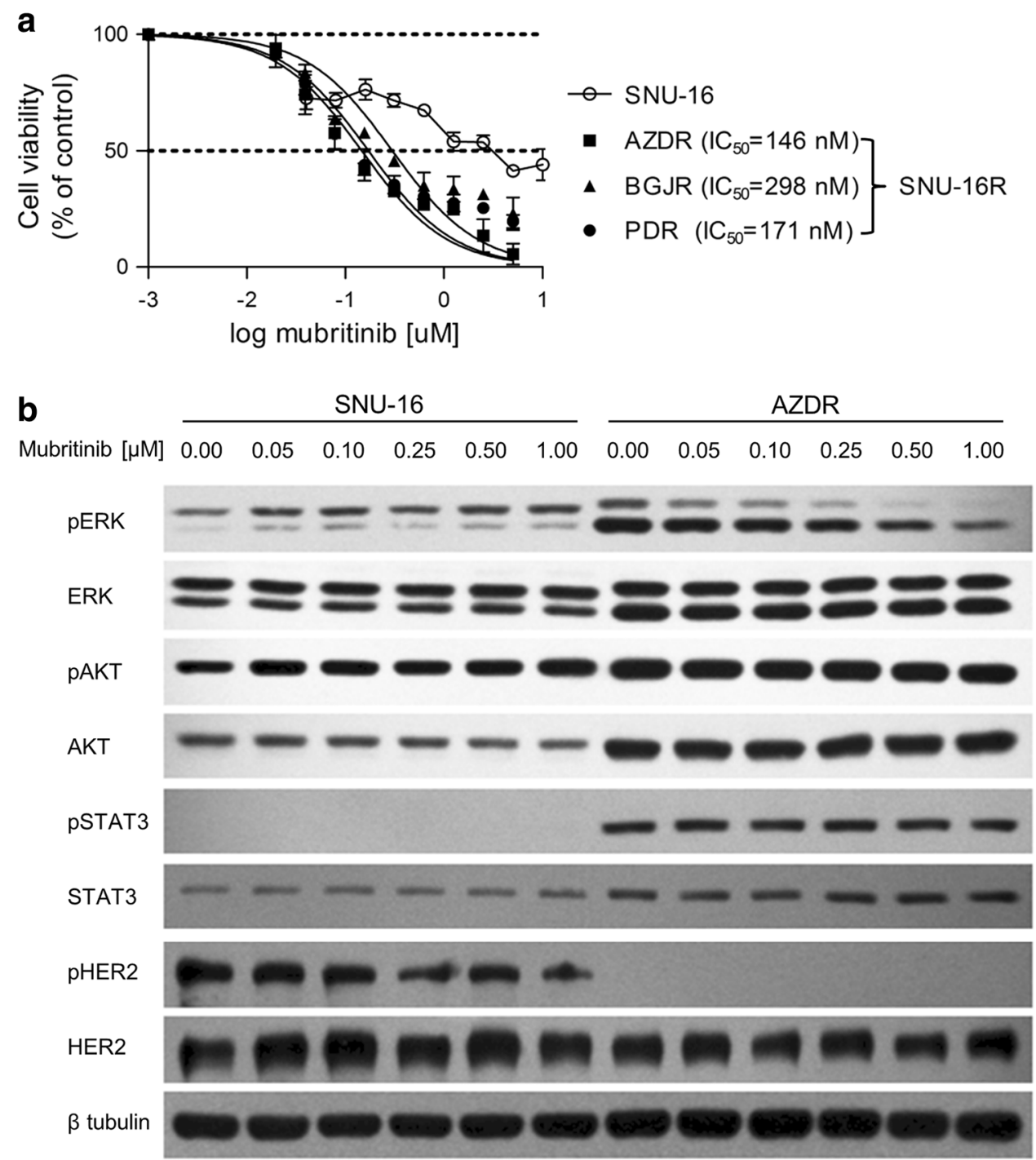

SNU-16 cells. These data suggest that mubritinib-mediated inhibition of SNU-16R cell proliferation might be the effect of suppression of ERK phosphorylation. Additionally, the activation of HER2 was markedly reduced in AZDR cells in comparison with wild-type SNU-16 cells (Figs. 2b, 5b). Thus, it is possible that the downregulation of pERK and substantial sensitivity of AZDR cells to mubritinib treatment are not the effect of HER2 inhibition. Furthermore, applying the HER2 inhibitor to SNU-16R cell lines for $48 \mathrm{~h}$ and $72 \mathrm{~h}$ did not reverse EMT since we did not observe an increase in E-cadherin level or a decrease in vimentin level (Fig. S1).

\section{Discussion}

To predict the mechanisms of resistance to selective FGFR inhibitors, we established three resistant cell lines-AZDR,
BGJR, and PDR - by long-term exposure of the human gastric cancer cell line SNU-16 to AZD4547, BGJ398, and PD173074, respectively. Compared with the parental SNU16 cells, the resistant sublines generated were less sensitive to the FGFR inhibitors tested, with approximately two orders of magnitude higher concentration being needed to inhibit cell viability by $50 \%$. Moreover, they displayed cross-resistance to other FGFR inhibitors. The morphological changes in resistant cells suggested EMT, which was further confirmed by a decrease of the level of the epithelial marker E-cadherin and an increase of the level of vimentin, a mesenchymal marker. It is important to note that AZD4547, BGJ398, or PD173074, three different FGFR inhibitors of diverse selectivity and physicochemical properties, induced the same EMT changes in SNU-16 cells. This suggests that the resistance described here is specific to FGFR inhibition and does not depend on the FGFR inhibitor itself. It is highly probable that the 
occurrence of the resistance associated with EMT depends on the cellular context since we did not observe EMT in UM-UC-14 bladder cell lines with FGFR3 mutation resistant to AZD4547, BGJ398, and PD173074, respectively (our unpublished data).

EMT refers to a complex molecular and cellular reorganization that in cancer is associated with metastasis, poor prognosis, and drug resistance. The changes from epithelial to mesenchymal status have been implicated in resistance to conventional and targeted anticancer drugs. It was confirmed that EMT induction confers resistance to EGFR inhibitors [26, 27], anaplastic lymphoma kinase inhibitors [34], and the multikinase inhibitor sorafenib [35]. Our study for the first time demonstrated that EMT is also a mechanism involved in acquired resistance to selective FGFR inhibitors in gastric cancer cells. At the time the manuscript was being written, Wang et al. [36] had discovered that acquired resistance to BGJ398 is characterized by EMT and a switch in dependency from FGFR to HER2/ HER3 in the FGFR3-dependent bladder cancer cell line RT112. There are also some reports in the literature concerning the correlation between epithelial/mesenchymal status and sensitivity to FGFR inhibitors; however, none of them refer to acquired resistance. The multitarget FGFR inhibitor dovitinib was shown to be more effective in bladder cancer cell lines with an epithelial phenotype [37]. Furthermore, PD173074 treatment induced mesenchymalto-epithelial transition in head and neck squamous carcinoma cells [38].

In our SNU-16-based resistance models the activation of EMT was associated with loss of FGFR2 expression. FGFR2 has previously been shown to be implicated in regulation of EMT. It was demonstrated that FGFR2IIIb, an isoform of FGFR2, occurs mainly in epithelial cells, in contrast to the FGFR2IIIc isoform, which is expressed in mesenchymal cells [39]. The switch from FGFR2IIIb to FGFR2IIIc was confirmed to promote an EMT phenotype [40]. Finally, loss of FGFR2IIIb was accompanied by loss of E-cadherin and acquisition of a mesenchymal phenotype in bladder carcinomas [41]. Our studies revealed that loss of FGFR2 expression is also implicated in resistanceassociated EMT induction in SNU-16 gastric cancer cells, in which the FGFR2IIIb isoform dominates [42]. In addition to loss of FGFR2 expression, we observed reduced activation/expression of other transmembrane receptors, such as MET, HER2, HER3, and EGFR. In our FGFRinhibitor-induced resistance models, the downregulation of these receptor kinases was accompanied by activation of SRC and downstream proteins such as ERK, AKT, and STAT3. Rho et al. [24] also observed reduced expression of EGFR, HER3, and MET and significantly enhanced activation of AKT in a gefitinib-resistant non-small-cell lung cancer cell line (A549) with EMT characteristics.
However, in contrast to our results, phosphorylation of ERK was downregulated in that model [24]. The loss of expression of receptors such as FGFR2, MET, and EGFR observed here in SNU-16R cell lines suggests that another pathway is responsible for activation of downstream proteins.

It was established that TGF- $\beta$ signaling is a key player in EMT-related chemotherapy and targeted therapy resistance in a number of malignancies [25, 43, 44]. TGF- $\beta$ activates its signaling via binding with high-affinity type II TGF- $\beta$ receptor (T $\beta R I I)$, which dimerizes with T $\beta R I$, which facilitates T $\beta$ RI phosphorylation. The activation of $T \beta R I$ leads to the propagation of signaling by the canonical SMAD-dependent pathway and the noncanonical SMADindependent pathway. The mesenchymal features and increase in phosphorylation level of proteins such as AKT, ERK, and SRC suggested activation of noncanonical TGF$\beta$ signaling in SNU-16R cell lines [45, 46]. However, we failed to induce EMT changes in parental SNU-16 cells by TGF- $\beta_{1}$ treatment. The only evidence of the induction of the TGF- $\beta$ signaling pathway in the resistant cells was upregulated TGF- $\beta$ ligand expression and slight activation of SMAD2. These differences between TGF- $\beta_{1}$-treated parental SNU-16 cells and SNU-16R cells suggest that, in addition to TGF- $\beta$-activated signaling, other unidentified pathways might be responsible for induction of an EMTlike phenotype of SNU-16R cells. A similar conclusion was drawn by Suda et al. [47], who also failed to prove the involvement of TGF- $\beta$ in acquired resistance to erlotinib. Moreover, applying SB525334 - an inhibitor of T $\beta R I-$ to SNU-16R cell lines neither reversed EMT nor reduced cell viability. Our results suggest that the application of a single inhibitor of one of the isoforms of T $\beta R I$ is not sufficient to reverse EMT-like changes in SNU-16R cell lines.

Since we did not observe any impact of the T $\beta R I$ inhibitor on the viability of the SNU-16R cell lines, we decided to test other known anticancer agents in order to determine an alternative therapeutic strategy for overcoming EMT-mediated resistance to selective FGFR inhibitors. We found that SNU-16R cells remained sensitive to the HSP90 inhibitor AUY922. In support of our results, HSP90 inhibitors effectively suppress growth of crizotinib-resistant H2228 lung cancer cells with EMT changes [34]. Kim et al. [34] suggested that the overcoming of resistance by HSP90 inhibitors resulted from reversal of EMT due to degradation of T $\beta R I I$ and further the restoration of E-cadherin expression. Finally, we found that an inhibitor of HER2 can overcome FGFR inhibitor resistance mediated by the EMT since all the SNU-16R cell lines, in contrast to parental cells, were highly sensitive to mubritinib. Moreover, the observed marked sensitivity of resistant cells to mubritinib suggests that use of this inhibitor might be an alternative therapeutic strategy for the 
management of gastric cancer resistant to FGFR inhibitors owing to EMT. The exact mechanism of mubritinib-mediated growth inhibition of the SNU-16R cell lines is unclear, and it might be the effect of inhibition of targets other than HER2, since we observed a decrease in activation of HER2 in SNU-16R cells, in contrast to results reported by Wang et al. [36]. Furthermore, unlike in the wild-type SNU-16 cells, mubritinib treatment of AZDR cells suppressed phosphorylation of ERK. However, further investigations are needed to clarify the mechanism of action of mubritinib in the SNU-16R cells. In conclusion, we found that EMT was induced as a result of long-term exposure of a gastric cancer cell line in vitro to selective FGFR inhibitors. Additionally, EMT induction was associated with reduction in expression of FGFR2 and other transmembrane receptors. On the basis of our study, EMT should be considered as a possible acquired resistance mechanism contributing to the decreased efficacy of FGFR-inhibitor-based therapy in cancer patients. Furthermore, our data suggest that the use of mubritinib or AUY922 treatment may be an alternative therapeutic approach for treating cancer patients with EMT-mediated resistance to FGFR inhibitors.

Grant Support This study was supported by Celon Pharma's own funds.

Conflict of interest Paulina Grygielewicz, Barbara Dymek, Anna Bujak, Pawel Gunerka, and Aleksandra Stanczak are full-time employees of Celon Pharma. Maciej Wieczorek is Chief Executive Officer of Celon Pharma, and Monika Lamparska-Przybysz, Karolina Dzwonek, and Daria Zdzalik were full-time employees of Celon Pharma during the experimental phase of this work. The authors declare that they have no conflict of interest.

Open Access This article is distributed under the terms of the Creative Commons Attribution License which permits any use, distribution, and reproduction in any medium, provided the original author(s) and the source are credited.

\section{References}

1. Eswarakumar VP, Lax I, Schlessinger J. Cellular signaling by fibroblast growth factor receptors. Cytokine Growth Factor Rev. 2005;16(2):139-49.

2. Kunii K, et al. FGFR2-amplified gastric cancer cell lines require FGFR2 and Erbb3 signaling for growth and survival. Cancer Res. 2008;68(7):2340-8.

3. Gru AA, Allred DC. FGFR1 amplification and the progression of non-invasive to invasive breast cancer. Breast Cancer Res. 2012;14(6): 116.

4. Billerey C, et al. Frequent FGFR3 mutations in papillary noninvasive bladder (pTa) tumors. Am J Pathol. 2001;158(6):1955-9.

5. Pollock PM, et al. Frequent activating FGFR2 mutations in endometrial carcinomas parallel germline mutations associated with craniosynostosis and skeletal dysplasia syndromes. Oncogene. 2007;26(50):7158-62.
6. Liao RG, et al. Inhibitor-sensitive FGFR2 and FGFR3 mutations in lung squamous cell carcinoma. Cancer Res. 2013;73(16): 5195-205.

7. Chesi $\mathrm{M}$, et al. Frequent translocation $\mathrm{t}(4 ; 14)(\mathrm{p} 16.3 ; \mathrm{q} 32.3)$ in multiple myeloma is associated with increased expression and activating mutations of fibroblast growth factor receptor 3 . Nat Genet. 1997;16(3):260-4.

8. Singh D, et al. Transforming fusions of FGFR and TACC genes in human glioblastoma. Science. 2012;337(6099):1231-5.

9. Dieci MV, et al. Fibroblast growth factor receptor inhibitors as a cancer treatment: from a biologic rationale to medical perspectives. Cancer Discov. 2013;3(3):264-79.

10. Mohammadi $\mathbf{M}$, et al. Crystal structure of an angiogenesis inhibitor bound to the FGF receptor tyrosine kinase domain. EMBO J. 1998;17(20):5896-904.

11. Gavine PR, et al. AZD4547: an orally bioavailable, potent, and selective inhibitor of the fibroblast growth factor receptor tyrosine kinase family. Cancer Res. 2012;72(8):2045-56.

12. Guagnano V, et al. Discovery of 3-(2,6-dichloro-3,5-dimethoxyphenyl)-1-\{6-[4-(4-ethyl-piperazin-1-yl)-phenylamino]-pyrimidin-4-yl -1-methyl-urea (NVP-BGJ398), a potent and selective inhibitor of the fibroblast growth factor receptor family of receptor tyrosine kinase. J Med Chem. 2011;54(20):7066-83.

13. Jemal A, et al. Global cancer statistics. CA Cancer J Clin. 2011;61(2):69-90.

14. Roukos DH. Targeting gastric cancer with trastuzumab: new clinical practice and innovative developments to overcome resistance. Ann Surg Oncol. 2010;17(1):14-7.

15. Zhao G, et al. A novel, selective inhibitor of fibroblast growth factor receptors that shows a potent broad spectrum of antitumor activity in several tumor xenograft models. Mol Cancer Ther. 2011;10(11):2200-10.

16. Guagnano V, et al. FGFR genetic alterations predict for sensitivity to NVP-BGJ398, a selective pan-FGFR inhibitor. Cancer Discov. 2012;2(12):1118-33.

17. Chell V, et al. Tumour cell responses to new fibroblast growth factor receptor tyrosine kinase inhibitors and identification of a gatekeeper mutation in FGFR3 as a mechanism of acquired resistance. Oncogene. 2013;32(25):3059-70.

18. Byron SA, et al. The $\mathrm{N} 550 \mathrm{~K} / \mathrm{H}$ mutations in FGFR2 confer differential resistance to PD173074, dovitinib, and ponatinib ATP-competitive inhibitors. Neoplasia. 2013;15(8):975-88.

19. Harbinski F, et al. Rescue screens with secreted proteins reveal compensatory potential of receptor tyrosine kinases in driving cancer growth. Cancer Discov. 2012;2(10):948-59.

20. Herrera-Abreu MT, et al. Parallel RNA interference screens identify EGFR activation as an escape mechanism in FGFR3mutant cancer. Cancer Discov. 2013;3(9):1058-71.

21. Bar-Am I, et al. Detection of amplified DNA sequences in human tumor cell lines by fluorescence in situ hybridization. Genes Chromosomes Cancer. 1992;4(4):314-20.

22. Xie L, et al. FGFR2 gene amplification in gastric cancer predicts sensitivity to the selective FGFR inhibitor AZD4547. Clin Cancer Res. 2013;19(9):2572-83.

23. Ku JL, Park JG. Biology of SNU cell lines. Cancer Res Treat. 2005;37(1):1-19.

24. Rho JK, et al. Epithelial to mesenchymal transition derived from repeated exposure to gefitinib determines the sensitivity to EGFR inhibitors in A549, a non-small cell lung cancer cell line. Lung Cancer. 2009;63(2):219-26.

25 . Brunen $\mathrm{D}$, et al. TGF- $\beta$ : an emerging player in drug resistance. Cell Cycle. 2013;12(18):2960-8.

26. Yauch RL, et al. Epithelial versus mesenchymal phenotype determines in vitro sensitivity and predicts clinical activity of erlotinib in lung cancer patients. Clin Cancer Res. 2005;11(24 Pt 1):8686-98. 
27. Thomson S, et al. Epithelial to mesenchymal transition is a determinant of sensitivity of non-small-cell lung carcinoma cell lines and xenografts to epidermal growth factor receptor inhibition. Cancer Res. 2005;65(20):9455-62.

28. Voulgari A, Pintzas A. Epithelial-mesenchymal transition in cancer metastasis: mechanisms, markers and strategies to overcome drug resistance in the clinic. Biochim Biophys Acta. 2009;1796(2):75-90.

29. Chaw SY, et al. Epithelial to mesenchymal transition (EMT) biomarkers - E-cadherin, beta-catenin, APC and vimentin - in oral squamous cell carcinogenesis and transformation. Oral Oncol. 2012;48(10):997-1006.

30. Kato $N$, et al. $\beta$-Catenin activation and epithelial-mesenchymal transition in the pathogenesis of pterygium. Invest Ophthalmol Vis Sci. 2007;48(4):1511-7.

31. Oft M, et al. TGF-beta1 and Ha-Ras collaborate in modulating the phenotypic plasticity and invasiveness of epithelial tumor cells. Genes Dev. 1996;10(19):2462-77.

32. Gal A, et al. Sustained TGF beta exposure suppresses Smad and non-Smad signalling in mammary epithelial cells, leading to EMT and inhibition of growth arrest and apoptosis. Oncogene. 2008;27(9):1218-30.

33. Kim YJ, et al. Transforming growth factor beta receptor I inhibitor sensitizes drug-resistant pancreatic cancer cells to gemcitabine. Anticancer Res. 2012;32(3):799-806.

34. Kim HR, et al. Epithelial-mesenchymal transition leads to crizotinib resistance in H2228 lung cancer cells with EML4-ALK translocation. Mol Oncol. 2013;7(6):1093-102.

35. van Malenstein $\mathrm{H}$, et al. Long-term exposure to sorafenib of liver cancer cells induces resistance with epithelial-to-mesenchymal transition, increased invasion and risk of rebound growth. Cancer Lett. 2013;329(1):74-83.

36. Wang $J$ et al. Ligand-associated ERBB2/3 activation confers acquired resistance to FGFR inhibition in FGFR3-dependent cancer cells. Oncogene. 2014. doi:10.1038/onc.2014.161.
37. Hanze J, et al. Epithelial mesenchymal transition status is associated with anti-cancer responses towards receptor tyrosinekinase inhibition by dovitinib in human bladder cancer cells. BMC Cancer. 2013;13:589.

38. Nguyen PT, et al. The FGFR1 inhibitor PD173074 induces mesenchymal-epithelial transition through the transcription factor AP-1. Br J Cancer. 2013;109(8):2248-58.

39. Warzecha CC, et al. ESRP1 and ESRP2 are epithelial cell-typespecific regulators of FGFR2 splicing. Mol Cell. 2009;33(5): 591-601.

40. Shirakihara T, et al. TGF- $\beta$ regulates isoform switching of FGF receptors and epithelial-mesenchymal transition. EMBO J. 2011;30(4):783-95.

41. De Medina SG, et al. Relationship between E-cadherin and fibroblast growth factor receptor $2 \mathrm{~b}$ expression in bladder carcinomas. Oncogene. 1999;18(41):5722-6.

42. Bai A, et al. GP369, an FGFR2-IIIb-specific antibody, exhibits potent antitumor activity against human cancers driven by activated FGFR2 signaling. Cancer Res. 2010;70(19):7630-9.

43. Huang S, et al. MED12 controls the response to multiple cancer drugs through regulation of TGF- $\beta$ receptor signaling. Cell. 2012;151(5):937-50.

44. Yao Z, et al. TGF- $\beta$ IL-6 axis mediates selective and adaptive mechanisms of resistance to molecular targeted therapy in lung cancer. Proc Natl Acad Sci U S A. 2010;107(35):15535-40.

45. Akhurst RJ, Hata A. Targeting the TGF $\beta$ signalling pathway in disease. Nat Rev Drug Discov. 2012;11(10):790-811.

46. Morrison CD, Parvani JG, Schiemann WP. The relevance of the TGF- $\beta$ paradox to EMT-MET programs. Cancer Lett. 2013;341(1):30-40.

47. Suda K, et al. Epithelial to mesenchymal transition in an epidermal growth factor receptor-mutant lung cancer cell line with acquired resistance to erlotinib. J Thorac Oncol. 2011;6(7):1152-61. 